\title{
Medical Image of the Month: Air Embolism in Transit
}

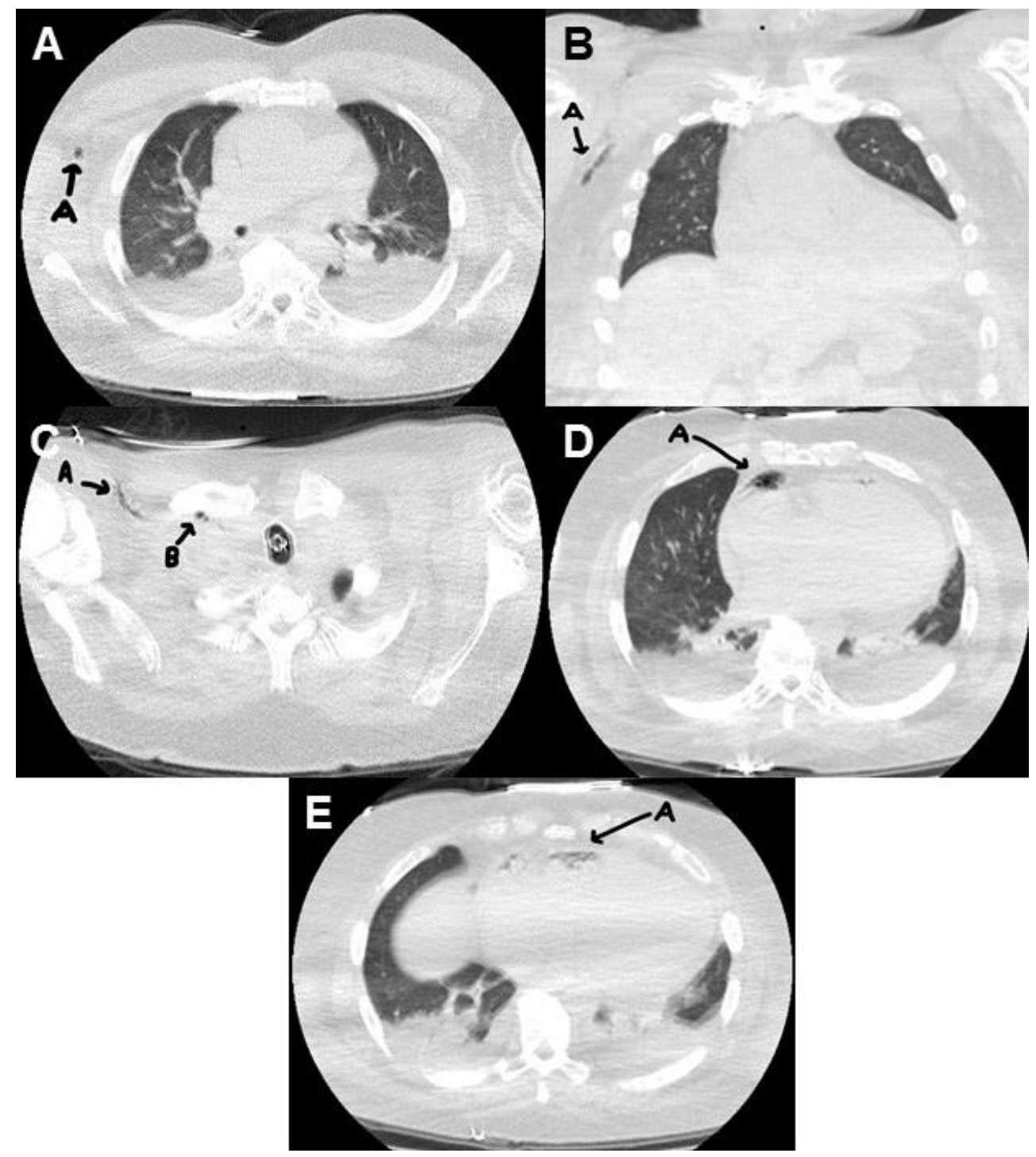

Figure 1. A: Axial CT of the chest without contrast in a lung window demonstrates air in the peripheral vein of the right upper extremity (arrow A). B: Coronal CT demonstrates air in the peripheral vein of right upper extremity (arrow A). C: Air in the right axillary vein (arrow $A$ ) and air in the right subclavian vein (arrow B). D: Air in the right atrium

(arrow A). E: air in the right ventricle (arrow A).

\section{Case Presentation}

Venous air embolism after fluid resuscitation with pressure infuser bag is rare but can occur without appropriate precautionary measures and can be fatal. We report a case of a 51-year-old male patient who presented to the emergency room (ER) with alcohol withdrawal, atrial fibrillation with rapid ventricular response, seizures, and massive 
aspiration leading to severe hypoxia and cardiac arrest. He was intubated, CPR was performed, and he received fluid resuscitation using the pressure infuser bag over the collapsible polypropylene (PP) based fluid bag through peripheral intravenous access in the dorsum of right hand. He was admitted to intensive care unit for further management after stabilization in the ER. Computed tomography (CT) of the chest without contrast was performed which showed air tracking along the peripheral vein in right upper extremity (Figure 1A,B) as well as in the right axillary and subclavian veins (Figure 1C). Air was also seen within the right atrium (Figure 1D) and right ventricle (Figure 1E) along with bilateral consolidative changes in the lower lobes.

Retrospectively, we discovered that the IV fluid bag was lying down on the bed during CT imaging, and the fluid bag had not been primed before placing it in the pressure infuser bag - both of which were the likely reasons for air entrapment. The patient was placed in left lateral decubitus (Durant's maneuver) and Trendelenburg position, given $100 \%$ supplemental oxygen, and provided with supportive care. A central line was placed in right internal jugular vein and blood was aspirated without any air. A transthoracic echocardiogram performed three days later showed no air in the right atrium or ventricle. A repeat CT of the chest without contrast performed seven days later demonstrated resolution of the air in the veins and right heart chambers. The patient improved from this episode, but unfortunately passed way from complications related to a large subdural hematoma.

\section{Discussion}

The risk of air embolism is less with collapsible fluid bags compared to glass or plastic bottles. However, there is still a risk of air entrapment with collapsible fluid bags without appropriate priming to remove small amounts of air present in the bag (1). Also, air can enter into the vascular system when the bag position is changed to a horizontal position from an upright position - which occurred in our case. Generally, a small amount of air in the venous system is absorbed without any major side effects but fatal consequences can occur with large amount of air (>3-5 ml/kg) and rapid air entry (2).

Naga S Sirikonda, MD, FCCP and Abdulmonam Ali, MD

Pulmonary and Critical Care

Good Samaritan Hospital, SSM Health

Mount Vernon, IL USA

\section{References}

1. Bakan M, Topuz U, Esen A, Basaranoglu G, Ozturk E. Inadvertent venous air embolism during cesarean section: Collapsible intravenous fluid bags without selfsealing outlet have risks. Case report. Braz J Anesthesiol. 2013 Jul-Aug;63(4):362-5. [CrossRef] [PubMed]

2. Shamim F, Abbasi S. Fatal vascular air embolism during fluid resuscitation as a complication of pressure infuser bag. J Emerg Trauma Shock. 2016 JanMar;9(1):46-7. [CrossRef] [PubMed] 